\title{
EKSISTENSI DAN KETUNGGALAN SOLUSI PERSAMAAN DIFERENSIAL LINIER ADVANCE-DELAY HOMOGEN
}

\author{
DEVI SILVIA RAHIMI \\ Fakultas Matematika dan Ilmu Pengetahuan Alam, Universitas Andalas \\ Limau Manis Padang, Sumatera Barat, Indonesia, \\ silvee2010@yahoo.com
}

\begin{abstract}
Abstrak. Pada tulisan ini akan dikonstruksi solusi persamaan diferensial linier advancedelay homogen. Selanjutnya, dengan menggunakan metode langkah (method of steps) dapat ditunjukkan bahwa suatu fungsi awal dengan syarat tertentu menjamin eksistensi dan ketunggalan solusinya. Tulisan ini mengeksplorasi kembali studi yang dilakukan oleh Ford dan Lumb (J. Compt. App. Math. 229, 2007).
\end{abstract}

Kata Kunci: Differential Equations, Initial Value Problem, heaviside

\section{Pendahuluan}

Persamaan diferensial advance-delay adalah salah satu kelas persamaan diferensial dimana turunannya bergantung pada variabel sebelumnya dan variabel sesudahnya. Persamaan ini juga dikenal dengan persamaan tipe campuran.

Kajian tentang persamaan ini awalnya dimotivasi oleh Chi, dkk pada tahun 1986 yang membahas aplikasi pada masalah konduksi saraf [1], Rustichini pada tahun 1987 yang membahas aplikasi dalam masalah kontrol optimal pada kasus autonomous linier dan untuk kasus nonlinier pada aplikasi dalam masalah dinamika ekonomi $[5,6]$.

Pada awal abad ini, kajian tentang persamaan diferensial advance-delay juga dibahas oleh Ford dan Lumb [2] untuk kasus homogen yang berbentuk

$$
x^{\prime}(t)=a x(t)+b x(t-1)+c x(t+1) .
$$

dimana $a, b, c, t \in \mathbb{R}$. Mereka menyimpulkan bahwa spesifikasi syarat batas tidak cukup untuk memastikan eksistensi solusi. Untuk kasus dimana solusinya ada, mereka memperkenalkan suatu algoritma numerik untuk menghitungnya. Selanjutnya, pendekatan ini dikembangkan lebih lanjut dalam $[7,8]$.

Pada tulisan ini, studi yang dilakukan oleh Ford dan Lumb akan dibahas kembali, yaitu untuk eksistensi dan ketunggalan solusi persamaan diferensial advancedelay (1.1).

\section{Eksistensi Solusi}

Sebelum membahas eksistensi solusinya, terlebih dahulu diberikan definisi dari solusi persamaan diferensial advance-delay. 
Definisi 2.1. [2] Solusi dari persamaan (1.1) pada interval $\left[t_{1}, t_{2}\right] \subseteq \mathbb{R}$ adalah fungsi kontinu $x:\left[t_{1}-1, t_{2}+1\right] \rightarrow \mathbb{C}$ yang sudah tentu kontinu pada $\left[t_{1}, t_{2}\right]$ dan yang memenuhi persamaan (1.1) untuk hampir setiap $t \in\left[t_{1}, t_{2}\right]$.

Dengan menggunakan transformasi $x(t) \rightarrow e^{a t} x(t)$ persamaan (1.1) dapat disederhanakan menjadi

$$
x^{\prime}(t)=\alpha x(t+1)+\beta x(t-1),
$$

dimana $\alpha=c e^{a}$ dan $\beta=b e^{-a}$.

Solusi persamaan (2.1) dengan $\alpha=\beta=1$ sudah pernah dibahas oleh Iakovleva [4] dengan menggunakan metode langkah (method of steps) yang memberikan suatu rumus iteratif. Metode tersebut akan diadopsi untuk kasus umum $\alpha, \beta \neq 0$.

Perhatikan kembali persamaan (2.1) dalam bentuk

$$
x(t)=\tilde{a} x^{\prime}(t-1)+\tilde{b} x(t-2)
$$

dimana $\tilde{a}=\frac{1}{\alpha}, \tilde{b}=\frac{-\beta}{\alpha}, t \in\left[t_{1}+1, t_{2}+1\right]$. Untuk membangun teorema eksistensi, misalkan $\alpha \neq 0, t_{1}=0, t_{2}=k-1, k \geq 2$ dan persamaan (2.2) sebagai masalah nilai awal dengan syarat awal $x(t)=\phi(t), t \in[-1,1]$, dimana

$$
\phi(t)=\left\{\begin{array}{l}
\phi_{1}(t), t \in[-1,0] \\
\phi_{2}(t), t \in[0,1] .
\end{array}\right.
$$

Untuk $t \in(1,2)$ diperoleh

$$
\begin{aligned}
x(t) & =\tilde{a} x^{\prime}(t-1)+\tilde{b} x(t-2) \\
& =\tilde{a} \phi_{2}^{\prime}(t-1)+\tilde{b} \phi_{1}(t-2) \\
& =\tilde{a} \phi^{\prime}(t-1)+\tilde{b} \phi(t-2) .
\end{aligned}
$$

Untuk $t \in(2,3)$ diperoleh

$$
\begin{aligned}
x(t) & =\tilde{a} x^{\prime}(t-1)+\tilde{b} x(t-2) \\
& =\tilde{a} \frac{d}{d t}\left(\tilde{a} \phi^{\prime}(t-2)+\tilde{b} \phi(t-3)\right)+\tilde{b} \phi_{2}(t-2) \\
& =\tilde{a}^{2} \phi^{\prime \prime}(t-2)+\tilde{a} \tilde{b} \phi^{\prime}(t-3)+\tilde{b} \phi(t-2) .
\end{aligned}
$$

Secara umum, untuk $t \in(2 l-1,2 l), l \in \mathbb{N}$, solusi $x(t)$ diberikan oleh

$$
x(t)=\sum_{k=0}^{l-1} \gamma_{l, 2 k} \tilde{a}^{2 k} \tilde{b}^{l-k} \phi^{(2 k)}(t-2 l)+\sum_{k=0}^{l-1} \gamma_{l, 2 k+1} \tilde{a}^{2 k+1} \tilde{b}^{l-k-1} \phi^{(2 k+1)}(t-(2 l-1)),
$$

sedangkan untuk $t \in(2 l, 2 l+1)$, solusi $x(t)$ diberikan oleh

$$
x(t)=\sum_{k=0}^{l} \delta_{l, 2 k} \tilde{a}^{2 k} \tilde{b}^{l-k} \phi^{(2 k)}(t-2 l)+\sum_{k=0}^{l-1} \delta_{l, 2 k+1} \tilde{a}^{2 k+1} \tilde{b}^{l-k} \phi^{(2 k+1)}(t-(2 l+1)),
$$


dimana $\gamma_{v, w}, \delta_{v, w}$ adalah koefisien (bilangan bulat) dengan $v, w \in \mathbb{N}$, didefinisikan untuk $l \geq 1$ sebagai berikut:

$$
\begin{aligned}
\gamma_{l, 2 k} & =\sum_{i=0}^{k} \gamma_{l-k-1+i, 2 i}+\sum_{i=0}^{k-1} \delta_{l-k-1+i, 2 i+1}, \\
\gamma_{l, 2 k+1} & =\sum_{i=0}^{k} \gamma_{l-k-1+i, 2 i}+\sum_{i=0}^{k} \delta_{l-k-1+i, 2 i+1} \\
\delta_{l, 2 k} & =\gamma_{l+1,2 k} \\
\delta_{l, 2 k+1} & =\gamma_{l, 2 k+1} .
\end{aligned}
$$

Perhatikan bahwa pada setiap interval, solusi $x(t)$ dinyatakan dengan peningkatan orde turunan dari fungsi $\phi$, sehingga perlu untuk mengasumsikan $\phi \in C^{\infty}[-1,1]$. Selanjutnya dapat ditunjukkan bahwa $\gamma_{l, 0}=1, \gamma_{l, 2 l-1}=1, \gamma_{l, 2 l-2}=1, \delta_{l, 0}=$ $1, \delta_{l, 2 l-1}=1, \delta_{l, 2 l}=1$, dan berlaku hubungan berikut:

$$
\begin{aligned}
\gamma_{p, 2 k-1}+\gamma_{p, 2 k} & =\gamma_{p+1,2 k}, \\
\gamma_{p+1,2 k}+\gamma_{p, 2 k+1} & =\gamma_{p+1,2 k+1} .
\end{aligned}
$$

Perlu diperhatikan bahwa $\gamma_{v, w}=\delta_{v, w}=0$ untuk $v<0$ atau $w<0$ dan $\gamma_{l, w}=$ $\delta_{l, w}=0$ untuk $w>2 l-1$.

Validitas dari persamaan (2.6) dan (2.7) dapat dibuktikan menggunakan induksi matematika dengan melakukan manipulasi aljabar. Perlu dicatat bahwa solusi bisa diperluas ke kiri dengan cara yang sama dengan menulis kembali persamaan tersebut dalam bentuk $x(t)=\tilde{a} x^{\prime}(t+1)+\tilde{b} x(t+2)$.

\section{Teorema Eksistensi dan Ketunggalan}

Teorema berikut memberikan syarat perlu dan syarat cukup bagi eksistensi solusi persamaan diferensial advance-delay homogen.

Teorema 3.1. [2] Solusi persamaan (2.1) dengan $\phi \in C^{\infty}[-1,1]$, ada dan diferensiabel jika dan hanya jika

$$
\phi^{(n+1)}(0)=\alpha \phi^{(n)}(1)+\beta \phi^{(n)}(-1),
$$

untuk $n=0,1,2, \cdots$.

Bukti. Karena $\phi \in C^{\infty}[-1,1]$ untuk setiap interval $(m, m+1), m \in \mathbb{Z}$, maka fungsi $x(t)$ ada dan diferensiabel tak hingga kali. Untuk membuktikan kekontinuan $x(t)$ dan eksistensi dari turunannya pada titik ujung $m$ dan $m+1$ (dan karenanya eksistensi dari $x(t)$ pada titik-titik tersebut) kita perlu untuk membuktikan persamaan

$$
x^{(i)}\left(m^{+}\right)=x^{(i)}\left(m^{-}\right), \quad i=0,1 \quad m=1,2, \cdots,
$$

dimana $x^{(i)}\left(m^{+}\right)$dan $x^{(i)}\left(m^{-}\right)$didefinisikan berturut-turut sebagai

$$
x^{(i)}\left(m^{+}\right)=\lim _{\epsilon \rightarrow 0^{+}} x^{(i)}(m+\epsilon), x^{(i)}\left(m^{-}\right)=\lim _{\epsilon \rightarrow 0^{+}} x^{(i)}(m-\epsilon) .
$$


Perhatikan bahwa $x\left(1^{-}\right)=\phi\left(1^{-}\right)=\phi(1)$ dan $\phi^{\prime}\left(0^{+}\right)=\phi^{\prime}(0)$. Dengan menggunakan induksi matematika akan dibuktikan

$$
x^{(i)}\left(m^{+}\right)=x^{(i)}\left(m^{-}\right), \quad i=0,1 \quad m=1,2, \cdots, k
$$

jika dan hanya jika

$$
\phi^{(n+1)}(0)=\alpha \phi^{(n)}(1)+\beta \phi^{(n)}(-1), \quad n=0,1,2, \cdots, k .
$$

Perhatikan terlebih dahulu untuk $k=1$.

(i) Akan ditunjukkan $x\left(1^{+}\right)=x\left(1^{-}\right) \Leftrightarrow \phi^{\prime}(0)=\alpha \phi(1)+\beta \phi(-1)$.

Dengan menggunakan (2.2), diperoleh

$$
x\left(1^{+}\right)=\tilde{a} x^{\prime}\left(0^{+}\right)+\tilde{b} x\left(-1^{+}\right) .
$$

Karena $x(t)=\phi(t), \phi \in C^{\infty}[-1,1]$, maka diperoleh

$$
\begin{aligned}
x\left(1^{+}\right)=x\left(1^{-}\right) & \Leftrightarrow x\left(1^{-}\right)=\tilde{a} \phi^{\prime}\left(0^{+}\right)+\tilde{b} \phi\left(-1^{+}\right) \\
& \Leftrightarrow \phi(1)=\tilde{a} \phi^{\prime}(0)+\tilde{b} \phi(-1) \\
& \Leftrightarrow \phi^{\prime}(0)=\frac{1}{\tilde{a}} \phi(1)-\frac{\tilde{b}}{\tilde{a}} \phi(-1) \\
& \Leftrightarrow \phi^{\prime}(0)=\alpha \phi(1)+\beta \phi(-1) .
\end{aligned}
$$

(ii) Akan ditunjukkan $x^{\prime}\left(1^{+}\right)=x^{\prime}\left(1^{-}\right) \Leftrightarrow \phi^{\prime \prime}(0)=\alpha \phi^{\prime}(1)+\beta \phi^{\prime}(-1)$.

Dengan menggunakan turunan pertama dari (2.2), diperoleh

$$
x^{\prime}(t)=\tilde{a} x^{\prime \prime}(t-1)+\tilde{b} x^{\prime}(t-2),
$$

sehingga

$$
x^{\prime}\left(1^{+}\right)=\tilde{a} x^{\prime \prime}\left(0^{+}\right)+\tilde{b} x^{\prime}\left(-1^{+}\right) .
$$

Karena $x(t)=\phi(t), \phi \in C^{\infty}[-1,1]$, maka diperoleh

$$
\begin{aligned}
x^{\prime}\left(1^{+}\right)=x^{\prime}\left(1^{-}\right) & \Leftrightarrow x^{\prime}\left(1^{-}\right)=\tilde{a} \phi^{\prime \prime}\left(0^{+}\right)+\tilde{b} \phi^{\prime}\left(-1^{+}\right) \\
& \Leftrightarrow \phi^{\prime}(1)=\tilde{a} \phi^{\prime \prime}(0)+\tilde{b} \phi^{\prime}(-1) \\
& \Leftrightarrow \phi^{\prime \prime}(0)=\frac{1}{\tilde{a}} \phi^{\prime}(1)-\frac{\tilde{b}}{\tilde{a}} \phi^{\prime}(-1) \\
& \Leftrightarrow \phi^{\prime \prime}(0)=\alpha \phi^{\prime}(1)+\beta \phi^{\prime}(-1) .
\end{aligned}
$$

Selanjutnya perhatikan kasus $k=2$.

(i) Akan ditunjukkan $x\left(2^{+}\right)=x\left(2^{-}\right) \Leftrightarrow \phi^{\prime \prime}(0)=\alpha \phi^{\prime}(1)+\beta \phi^{\prime}(-1)$. Dengan menggunakan (2.7) untuk $l=1$, diperoleh

$$
\begin{aligned}
x\left(2^{+}\right) & =\sum_{k=0}^{1}\left(\delta_{1,2 k} \tilde{a}^{2 k} \tilde{b}^{1-k} \phi^{(2 k)}\left(0^{+}\right)\right)+\delta_{1,1} \tilde{a}^{1} \tilde{b} \phi^{\prime}\left(-1^{+}\right) \\
& =\tilde{b} \phi\left(0^{+}\right)+\tilde{a}^{2} \phi^{\prime \prime}\left(0^{+}\right)+\tilde{a} \tilde{b} \phi^{\prime}\left(-1^{+}\right) .
\end{aligned}
$$

Kemudian dengan menggunakan (2.6) untuk $l=1$, diperoleh

$$
\begin{aligned}
x\left(2^{-}\right) & =\gamma_{1,0} \tilde{b} \phi\left(0^{-}\right)+\gamma_{1,1} \tilde{a} \phi^{\prime}\left(1^{-}\right) \\
& =\tilde{b} \phi\left(0^{-}\right)+\tilde{a} \phi^{\prime}\left(1^{-}\right) .
\end{aligned}
$$


Karena $\phi \in C^{\infty}[-1,1]$, maka berlaku

$$
\begin{aligned}
x\left(2^{+}\right)=x\left(2^{-}\right) & \Leftrightarrow \tilde{b} \phi(0)+\tilde{a}^{2} \phi^{\prime \prime}(0)+\tilde{a} \tilde{b} \phi^{\prime}(-1)=\tilde{b} \phi(0)+\tilde{a} \phi^{\prime}(1) \\
& \Leftrightarrow \tilde{a}^{2} \phi^{\prime \prime}(0)=\tilde{a} \phi^{\prime}(1)-\tilde{a} \tilde{b} \phi^{\prime}(-1) \\
& \Leftrightarrow \phi^{\prime \prime}(0)=\frac{1}{\tilde{a}} \phi^{\prime}(1)-\frac{\tilde{b}}{\tilde{a}} \phi^{\prime}(-1) \\
& \Leftrightarrow \phi^{\prime \prime}(0)=\alpha \phi^{\prime}(1)+\beta \phi^{\prime}(-1) .
\end{aligned}
$$

(ii) Akan ditunjukkan $x^{\prime}\left(2^{+}\right)=x^{\prime}\left(2^{-}\right) \Leftrightarrow \phi^{\prime \prime \prime}(0)=\alpha \phi^{\prime \prime}(1)+\beta \phi^{\prime \prime}(-1)$.

Dengan menggunakan turunan pertama dari (2.7) untuk $l=1$, maka

$$
\begin{aligned}
x^{\prime}\left(2^{+}\right) & =\sum_{k=0}^{1}\left(\delta_{1,2 k} \tilde{a}^{2 k} \tilde{b}^{1-k} \phi^{(2 k+1)}\left(0^{+}\right)\right)+\delta_{1,1} \tilde{a} \tilde{b} \phi^{\prime \prime}\left(-1^{+}\right) \\
& =\tilde{b} \phi\left(0^{+}\right)+\tilde{a}^{2} \phi^{\prime \prime \prime}\left(0^{+}\right)+\tilde{a} \tilde{b} \phi^{\prime \prime}\left(-1^{+}\right)
\end{aligned}
$$

dan dengan menggunakan turunan pertama dari (2.6) untuk $l=1$, maka diperoleh

$$
\begin{aligned}
x^{\prime}\left(2^{-}\right) & =\gamma_{1,0} \tilde{b} \phi^{\prime}\left(0^{-}\right)+\gamma_{1,1} \tilde{a} \phi^{\prime \prime}\left(1^{-}\right) \\
& =\tilde{b} \phi^{\prime}\left(0^{-}\right)+\tilde{a} \phi^{\prime \prime}\left(1^{-}\right) .
\end{aligned}
$$

Karena $\phi \in C^{\infty}[-1,1]$, maka berlaku

$$
\begin{aligned}
x^{\prime}\left(2^{+}\right)=x^{\prime}\left(2^{-}\right) & \Leftrightarrow \tilde{b} \phi^{\prime}(0)+\tilde{a}^{2} \phi^{\prime \prime \prime}(0)+\tilde{a} \tilde{b} \phi^{\prime}(-1)=\tilde{b} \phi^{\prime}(0)+\tilde{a} \phi^{\prime \prime}(1) \\
& \Leftrightarrow \tilde{a}^{2} \phi^{\prime \prime \prime}(0)=\tilde{a} \phi^{\prime \prime}(1)-\tilde{a} \tilde{b} \phi^{\prime \prime}(-1) \\
& \Leftrightarrow \phi^{\prime \prime \prime}(0)=\frac{1}{\tilde{a}} \phi^{\prime \prime}(1)-\frac{\tilde{b}}{\tilde{a}} \phi^{\prime \prime}(-1) \\
& \Leftrightarrow \phi^{\prime \prime \prime}(0)=\alpha \phi^{\prime \prime}(1)+\beta \phi^{\prime \prime}(-1) .
\end{aligned}
$$

Sekarang andaikan benar untuk $k=2 l-1$, yaitu berlaku

$$
\begin{aligned}
x\left((2 l-1)^{+}\right) & =x\left((2 l-1)^{-}\right) \Leftrightarrow \phi^{(2 l-1)}(0)=\alpha \phi^{(2 l-2)}(1)+\beta \phi^{(2 l-2)}(-1), \\
x^{\prime}\left((2 l-1)^{+}\right) & =x^{\prime}\left((2 l-1)^{-}\right) \Leftrightarrow \phi^{(2 l)}(0)=\alpha \phi^{(2 l-1)}(1)+\beta \phi^{(2 l-1)}(-1) .
\end{aligned}
$$

Akan ditunjukkan bahwa pernyataan tersebut benar untuk $k=2 l$.

(i) Akan ditunjukkan

$$
x\left(2 l^{+}\right)=x\left(2 l^{-}\right) \Leftrightarrow \phi^{(2 l)}(0)=\alpha \phi^{(2 l-1)}(1)+\beta \phi^{(2 l-1)}(-1) .
$$

Perhatikan bahwa

$$
\begin{aligned}
x\left(2 l^{+}\right) & =x\left(2 l^{-}\right) \\
& \left.\Leftrightarrow \sum_{k=0}^{l} \delta_{l, 2 k} \tilde{a}^{2 k} \tilde{b}^{l-k} \phi^{(2 k)}\left(0^{+}\right)+\sum_{k=0}^{l-1} \delta_{l, 2 k+1} \tilde{a}^{2 k+1} \tilde{b}^{l-k} \phi^{(2 k+1)}\left(-1^{+}\right)\right) \\
& =\sum_{k=0}^{l-1}\left(\gamma_{l, 2 k} \tilde{a}^{2 k} \tilde{b}^{l-k} \phi^{(2 k)}\left(0^{-}\right)+\gamma_{l, 2 k+1} \tilde{a}^{2 k+1} \tilde{b}^{l-k-1} \phi^{(2 k+1)}\left(1^{-} \chi \xi\right) .9\right)
\end{aligned}
$$


Selanjutnya, persamaan (3.10) menjadi

$$
\begin{aligned}
& x\left(2 l^{+}\right)=x\left(2 l^{-}\right) \\
\Leftrightarrow & \left.\sum_{k=0}^{l} \gamma_{l+1,2 k} \tilde{a}^{2 k} \tilde{b}^{l-k} \phi^{(2 k)}\left(0^{+}\right)+\sum_{k=0}^{l-1} \gamma_{l, 2 k+1} \tilde{a}^{2 k+1} \tilde{b}^{l-k} \phi^{(2 k+1)}\left(-1^{+}\right)\right) \\
= & \left.\sum_{k=0}^{l-1}\left(\gamma_{l, 2 k} \tilde{a}^{2 k} \tilde{b}^{l-k} \phi^{(2 k)}\left(0^{-}\right)+\gamma_{l, 2 k+1} \tilde{a}^{2 k+1} \tilde{b}^{l-k-1} \phi^{(2 k+1)}\left(1^{-}\right)\right)\right) \\
\Leftrightarrow & \gamma_{l+1,2 l} \tilde{a}^{2 l} \phi^{(2 l)}\left(0^{+}\right)+\sum_{k=0}^{l-1}\left[\gamma_{l+1,2 k}-\gamma_{l, 2 k}\right] \tilde{a}^{2 k} \tilde{b}^{l-k} \phi^{(2 k)}(0) \\
= & \left.\left.\sum_{k=0}^{l-1} \gamma_{l, 2 k+1} \tilde{a}^{2 k+1} \tilde{b}^{l-k-1}\left[\phi^{(2 k+1)}\left(1^{-}\right)\right)-\tilde{b} \phi^{(2 k+1)}\left(-1^{+}\right)\right)\right] .
\end{aligned}
$$

Misalkan $r=k+1$, maka

$$
\begin{aligned}
& x\left(2 l^{+}\right)=x\left(2 l^{-}\right) \\
\Leftrightarrow & \gamma_{l+1,2 l} \tilde{a}^{2 l} \phi^{(2 l)}\left(0^{+}\right)+\sum_{k=0}^{l-1} \gamma_{l, 2 k-1} \tilde{a}^{2 k} \tilde{b}^{l-k}\left[\frac{1}{\tilde{a}} \phi^{(2 k-1)}(1)-\frac{\tilde{b}}{\tilde{a}} \phi^{(2 k-1)}(-1)\right] \\
= & \left.\left.\sum_{r=1}^{l} \gamma_{l, 2 r-1} \tilde{a}^{2 r-1} \tilde{b}^{l-r}\left[\phi^{(2 r-1)}\left(1^{-}\right)\right)-\tilde{b} \phi^{(2 r-1)}\left(-1^{+}\right)\right)\right] \\
\Leftrightarrow & \gamma_{l+1,2 l} \tilde{a}^{2 l} \phi^{(2 l)}\left(0^{+}\right)+\sum_{k=0}^{l-1} \gamma_{l, 2 k-1} \tilde{a}^{2 k-1} \tilde{b}^{l-k}\left[\phi^{(2 k-1)}(1)-\tilde{b} \phi^{(2 k-1)}(-1)\right] \\
= & \left.\left.\sum_{k=1}^{l} \gamma_{l, 2 k-1} \tilde{a}^{2 k-1} \tilde{b}^{l-k}\left[\phi^{(2 k-1)}\left(1^{-}\right)\right)-\tilde{b} \phi^{(2 k-1)}\left(-1^{+}\right)\right)\right] \\
\Leftrightarrow & \gamma_{l+1,2 l} \tilde{a}^{2 l} \phi^{(2 l)}\left(0^{+}\right)+\gamma_{l,-1} \tilde{a}^{-1} \tilde{b}^{l}\left[\phi^{(-1)}(1)-\tilde{b} \phi^{(-1)}(-1)\right] \\
= & \left.\left.\gamma_{l, 2 l-1} \tilde{a}^{2 l-1}\left[\phi^{(2 l-1)}\left(1^{-}\right)\right)-\tilde{b} \phi^{(2 l-1)}\left(-1^{+}\right)\right)\right]
\end{aligned}
$$

Perhatikan bahwa $\gamma_{l+1,2 l}=\gamma_{l, 2 l-1}=1$ dan $\gamma_{l,-1}=0$, sehingga diperoleh

$$
\begin{aligned}
& x\left(2 l^{+}\right)=x\left(2 l^{-}\right) \\
\Leftrightarrow & \left.\left.\tilde{a}^{2 l} \phi^{(2 l)}\left(0^{+}\right)=\tilde{a}^{2 l-1}\left[\phi^{(2 l-1)}\left(1^{-}\right)\right)-\tilde{b} \phi^{(2 l-1)}\left(-1^{+}\right)\right)\right] \\
\Leftrightarrow & \left.\left.\phi^{(2 l)}\left(0^{+}\right)=\frac{1}{\tilde{a}}\left[\phi^{(2 l-1)}\left(1^{-}\right)\right)-\tilde{b} \phi^{(2 l-1)}\left(-1^{+}\right)\right)\right] \\
\Leftrightarrow & \phi^{(2 l)}(0)=\alpha \phi^{(2 l-1)}(1)+\beta \phi^{(2 l-1)}(-1) .
\end{aligned}
$$

(ii) Akan ditunjukkan $x^{\prime}\left(2 l^{+}\right)=x^{\prime}\left(2 l^{-}\right) \Leftrightarrow \phi^{(2 l+1)}(0)=\alpha \phi^{(2 l)}(1)+\beta \phi^{(2 l)}(-1)$. 
Perhatikan bahwa

$$
\begin{aligned}
& x^{\prime}\left(2 l^{+}\right)=x^{\prime}\left(2 l^{-}\right) \\
\Leftrightarrow & \left.\sum_{k=0}^{l} \delta_{l, 2 k} \tilde{a}^{2 k} \tilde{b}^{l-k} \phi^{(2 k+1)}\left(0^{+}\right)+\sum_{k=0}^{l-1} \delta_{l, 2 k+1} \tilde{a}^{2 k+1} \tilde{b}^{l-k} \phi^{(2 k+2)}\left(-1^{+}\right)\right) \\
= & \left.\sum_{k=0}^{l-1} \gamma_{l, 2 k} \tilde{a}^{2 k} \tilde{b}^{l-k} \phi^{(2 k+1)}\left(0^{-}\right)+\gamma_{l, 2 k+1} \tilde{a}^{2 k+1} \tilde{b}^{l-k-1} \phi^{(2 k+2)}\left(1^{-}\right)\right)
\end{aligned}
$$

Selanjutnya,

$$
\begin{aligned}
& x^{\prime}\left(2 l^{+}\right)=x^{\prime}\left(2 l^{-}\right) \\
\Leftrightarrow & \left.\sum_{k=0}^{l} \gamma_{l+1,2 k} \tilde{a}^{2 k} \tilde{b}^{l-k} \phi^{(2 k+1)}\left(0^{+}\right)+\sum_{k=0}^{l-1} \gamma_{l, 2 k+1} \tilde{a}^{2 k+1} \tilde{b}^{l-k} \phi^{(2 k+2)}\left(-1^{+}\right)\right) \\
= & \left.\sum_{k=0}^{l-1}\left[\gamma_{l, 2 k} \tilde{a}^{2 k} \tilde{b}^{l-k} \phi^{(2 k+1)}\left(0^{-}\right)+\gamma_{l, 2 k+1} \tilde{a}^{2 k+1} \tilde{b}^{l-k-1} \phi^{(2 k+2)}\left(1^{-}\right)\right)\right] \\
\Leftrightarrow & \gamma_{l+1,2 l} \tilde{a}^{2 l} \phi^{(2 l+1)}\left(0^{+}\right)+\sum_{k=0}^{l-1}\left[\gamma_{l+1,2 k}-\gamma_{l, 2 k}\right] \tilde{a}^{2 k} \tilde{b}^{l-k} \phi^{(2 k+1)}(0) \\
= & \left.\left.\sum_{k=0}^{l-1} \gamma_{l, 2 k+1} \tilde{a}^{2 k+1} \tilde{b}^{l-k-1}\left[\phi^{(2 k+2)}\left(1^{-}\right)\right)-\tilde{b} \phi^{(2 k+2)}\left(-1^{+}\right)\right)\right] \\
\Leftrightarrow & \gamma_{l+1,2 l} \tilde{a}^{2 l} \phi^{(2 l+1)}\left(0^{+}\right)+\sum_{k=0}^{l-1} \gamma_{l, 2 k-1} \tilde{a}^{2 k} \tilde{b}^{l-k}\left[\frac{1}{\tilde{a}} \phi^{2 k}(1)-\frac{\tilde{b}}{\tilde{a}} \phi^{2 k}(-1)\right] \\
= & \left.\left.\sum_{k=0}^{l-1} \gamma_{l, 2 k+1} \tilde{a}^{2 k+1} \tilde{b}^{l-k-1}\left[\phi^{(2 k+2)}\left(1^{-}\right)\right)-\tilde{b} \phi^{(2 k+2)}\left(-1^{+}\right)\right)\right] .
\end{aligned}
$$

Misalkan $r=k+1$, maka

$$
\begin{aligned}
& x^{\prime}\left(2 l^{+}\right)=x^{\prime}\left(2 l^{-}\right) \\
\Leftrightarrow & \gamma_{l+1,2 l} \tilde{a}^{2 l} \phi^{(2 l+1)}\left(0^{+}\right)+\sum_{k=0}^{l-1} \gamma_{l, 2 k-1} \tilde{a}^{2 k-1} \tilde{b}^{l-k}\left[\phi^{2 k}(1)-\tilde{b} \phi^{2 k}(-1)\right] \\
= & \left.\left.\sum_{r=1}^{l} \gamma_{l, 2 r-1} \tilde{a}^{2 r-1} \tilde{b}^{l-r}\left[\phi^{(2 r)}\left(1^{-}\right)\right)-\tilde{b} \phi^{(2 r)}\left(-1^{+}\right)\right)\right] .
\end{aligned}
$$

Karena $r$ hanyalah variabel dummy, maka persamaan terakhir menjadi

$$
\begin{aligned}
& x^{\prime}\left(2 l^{+}\right)=x^{\prime}\left(2 l^{-}\right) \\
\Leftrightarrow & \gamma_{l+1,2 l} \tilde{a}^{2 l} \phi^{(2 l+1)}\left(0^{+}\right)+\sum_{k=0}^{l-1} \gamma_{l, 2 k-1} \tilde{a}^{2 k-1} \tilde{b}^{l-k}\left[\phi^{2 k}(1)-\tilde{b} \phi^{2 k}(-1)\right] \\
= & \left.\left.\sum_{k=1}^{l} \gamma_{l, 2 k-1} \tilde{a}^{2 k-1} \tilde{b}^{l-k}\left[\phi^{(2 k)}\left(1^{-}\right)\right)-\tilde{b} \phi^{(2 k)}\left(-1^{+}\right)\right)\right]
\end{aligned}
$$




$$
\begin{aligned}
\Leftrightarrow & \gamma_{l+1,2 l} \tilde{a}^{2 l} \phi^{(2 l+1)}\left(0^{+}\right)+\gamma_{l,-1} \tilde{a}^{-1} \tilde{b}^{l}[\phi(1)-\tilde{b} \phi(-1)] \\
& \left.\left.=\gamma_{l, 2 l-1} \tilde{a}^{2 l-1}\left[\phi^{(2 l)}\left(1^{-}\right)\right)-\tilde{b} \phi^{(2 l)}\left(-1^{+}\right)\right)\right] \\
\Leftrightarrow & \tilde{a} \phi^{(2 l+1)}\left(0^{+}\right)=\phi^{(2 l)}\left(1^{-}\right)-\tilde{b} \phi^{(2 l)}\left(-1^{+}\right) \\
\Leftrightarrow & \phi^{(2 l+1)}(0)=\alpha \phi^{(2 l)}(1)+\beta \phi^{(2 l)}(-1)
\end{aligned}
$$

Jadi, teorema terbukti.

Selanjutnya syarat cukup bagi ketunggalan solusi dari persamaan diferensial advance-delay homogen diberikan oleh teorema berikut.

Teorema 3.2. [2] Misalkan $\phi \in C^{\infty}[-1,1]$. Jika solusi $x(t)$ dari persamaan (2.1) ada dan diferensiabel, maka solusinya tunggal.

Bukti. Pada interval buka $(m, m+1)$, solusi dari persamaan (2.1) diberikan oleh persamaan (2.6) dan (2.7) yang jelas terdefinisi secara tunggal. Pada titik-titik ujungnya, solusi tersebut juga merupakan solusi tunggal karena kekontinuan dari $x(t)$ sebagaimana yang telah dibuktikan oleh Teorema 3.1.

\section{Ucapan Terima Kasih}

Penulis mengucapkan terima kasih kepada Bapak Dr. Mahdhivan Syafwan, Ibu Dr. Yanita, Bapak Prof. Dr. Syafrizal Sy, Bapak Dr. Admi Nazra, Ibu Dr. Susila Bahri dan Bapak Dr. Muhafzan yang telah memberikan masukan dan saran, sehingga tulisan ini dapat diselesaikan dengan baik.

\section{Daftar Pustaka}

[1] Chi, Henjin., Jonathan, Bell dan Brian, Hassard. 1986. Numerical Solution of A Nonlinear Advance-Delay-Differential Equation from Nerve Conduction Theory. J. Math Biol, 4: 583 - 601.

[2] Ford, N.J. dan Lumb, P.M. 2007. Mixed-Type Functional Differential Equations: A Numerical Approach. Journal of Computational and Applied Mathematics, 229: 471 - 479 .

[3] Ford, N.J. dan Lumb, P.M. 2007. Mixed-Type Functional Differential Equations: A Numerical Approach (extended version). Report UCM, Department of Mathematics, Univerity of Chester.

[4] Iakovleva, V. dan Carmen, J.V. 2015. On The Solution of Differential Equations with Delayed and Advanced Arguments. Electronic Journal of Differential Equations, 13 : 57 - 63.

[5] Rustichini, A. 1989. Functional Differential Equations of Mixed Type. Journal of Dynamics and Differential Equations, 1: 121 - 143.

[6] Rustichini, A. 1989. Hopf Bifurcation for Functional Differential Equations of Mixed Type. Journal of Dynamics and Differential Equations, 1: 145 - 177.

[7] Teodoro, F, dkk. 2009. New Approach to The Numerical Solution of ForwardBackward Equations. Front Math China, 4(1) : 155 - 168.

[8] Teodoro, F, dkk. 2008. Numerical Modelling of A Functional Differential Equation with Deviating Arguments Using A Collocation Method. AIP Proceedings, 1048: 553 - 557 